\title{
Interventions for preventing and managing radiation-induced skin reactions in cancer patients (Protocol)
}

\author{
Chan R, Webster J, Battistutta D, Chung B, Brooks L
}

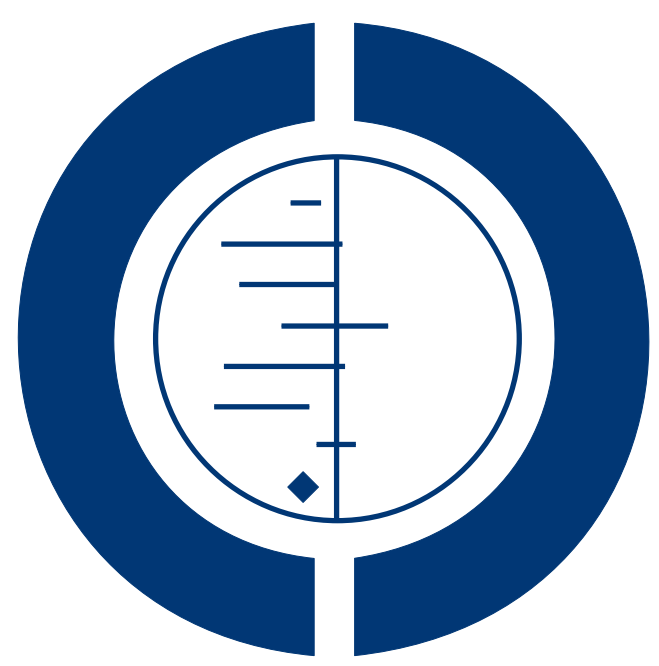

\section{THE COCHRANE COLLABORATION $^{\circledR}$}

This is a reprint of a Cochrane protocol, prepared and maintained by The Cochrane Collaboration and published in The Cochrane Library 2010, Issue 5

http://www.thecochranelibrary.com

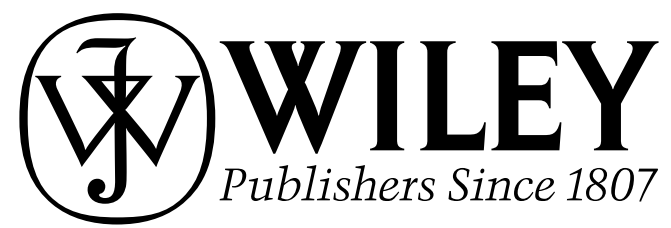

Interventions for preventing and managing radiation-induced skin reactions in cancer patients (Protocol)

Copyright (C) 2010 The Cochrane Collaboration. Published by John Wiley \& Sons, Ltd. 
TABLE OF CONTENTS

HEADER . . . . . . . . . . . . . . . . . . . . . . . . . . . . . . . . . . . . . . . . . 1

ABSTRACT . . . . . . . . . . . . . . . . . . . . . . . . . . . . . . . . . . . . . . 1

BACKGROUND . . . . . . . . . . . . . . . . . . . . . . . . . . . . . . . . . . . . . . . . . $\quad 2$

Figure 1. . . . . . . . . . . . . . . . . . . . . . . . . . . . . . . . . . . . . 2

OBJECTIVES . . . . . . . . . . . . . . . . . . . . . . . . . . . . . . . . . . . . . . . . .

METHODS . . . . . . . . . . . . . . . . . . . . . . . . . . . . . . . . . . . . . . $\quad 3$

ACKNOWLEDGEMENTS . . . . . . . . . . . . . . . . . . . . . . . . . . . . . . . . . . . . . . $\quad$. 7

REFERENCES . . . . . . . . . . . . . . . . . . . . . . . . . . . . . . . . . . . . . . . . . . . 8

APPENDICES . . . . . . . . . . . . . . . . . . . . . . . . . . . . . . . . . . . . . 9

HISTORY . . . . . . . . . . . . . . . . . . . . . . . . . . . . . . . . . . . . . . . . . $\quad 10$

CONTRIBUTIONS OF AUTHORS . . . . . . . . . . . . . . . . . . . . . . . . . . . . . . . . . . . . . . . . . .

DECLARATIONS OF INTEREST . . . . . . . . . . . . . . . . . . . . . . . . . . . . . . . . . . . . $\quad . \quad 10$

SOURCES OF SUPPORT . . . . . . . . . . . . . . . . . . . . . . . . . . . . . . . . . . . . . . . . . . 10 


\title{
[Intervention Protocol] \\ Interventions for preventing and managing radiation-induced skin reactions in cancer patients
}

\author{
Raymond Chan ${ }^{1}$, Joan Webster ${ }^{2}$, Diana Battistutta ${ }^{3}$, Bryan Chung ${ }^{4}$, Lance Brooks ${ }^{5}$ \\ ${ }^{1}$ Cancer Care Services, Royal Brisbane and Women's Hospital, Herston, Australia; and, School of Nursing and Midwifery, Queensland \\ University of Technology, Kelvin Grove, Australia. ${ }^{2}$ Centre for Clinical Nursing, Royal Brisbane and Women's Hospital, Herston, \\ Australia. ${ }^{3}$ Research Methods Group, Institute of Health and Biomedical Innovation, Queensland University of Technology (QUT), \\ Brisbane, Australia. ${ }^{4}$ Division of Plastic Surgery, QEII Health Science Centre, Halifax, Canada. ${ }^{5}$ Cancer Voices Queensland, Spring \\ Hill, Brisbane, Queensland, Australia \\ Contact address: Raymond Chan, Cancer Care Services, Royal Brisbane and Women's Hospital, Herston, Australia; and, School of \\ Nursing and Midwifery, Queensland University of Technology, Kelvin Grove, QLD, Australia. raymond_chan@health.qld.gov.au.
}

Editorial group: Cochrane Skin Group.

Publication status and date: New, published in Issue 5, 2010.

Citation: Chan R, Webster J, Battistutta D, Chung B, Brooks L. Interventions for preventing and managing radiation-induced skin reactions in cancer patients. Cochrane Database of Systematic Reviews 2010, Issue 5. Art. No.: CD008522. DOI: 10.1002/14651858.CD008522.

Copyright (C) 2010 The Cochrane Collaboration. Published by John Wiley \& Sons, Ltd.

\section{A B S T R A C T}

This is the protocol for a review and there is no abstract. The objectives are as follows:

To assess the effects of any interventions which aim to prevent or manage radiation-induced skin reactions in people with cancer. 


\section{B A C K G R O U N D}

\section{Description of the condition}

Radiation therapy remains an essential treatment for people with cancer and is associated with a number of short-term and longterm side-effects (Maduro 2003; Vaz 2007). One of these sideeffects is radiation-induced skin reaction (RISR), affecting up to $95 \%$ of people receiving radiation therapy for their cancer (Porock 2002). The reactions are a result of radiation treatment disrupting the normal process of cell division and regeneration, resulting in cell damage or cell death (Sitton 1992). The damage can be a result of several processes, including a reduction of endothelial cell changes, inflammation, and epidermal cell death (Hymes 2006). Radiation-induced skin reactions are often characterised by swelling, redness, pigmentation, fibrosis, and ulceration of the skin. Signs and symptoms are expressed as pain, warmth, burning, and itching of the skin (Noble-Adams 1999). The development of
RISR may occur two to three weeks after treatment commences, and may persist up to four weeks after the treatment ends (Naylor 2001). On rare occasions, an acute reaction called "radiation recall dermatitis" may also occur. The phenomenon of radiation recall dermatitis is a reaction that may occur after the administration of certain drugs days or in some cases years after exposure to ionising radiation (Camidge 2001).

The factors influencing the development or severity of RISR have been classified in the literature as either being intrinsic or extrinsic (Glean 2001). Intrinsic factors include age, general health, ethnic origin, coexisting diseases, UV exposure, hormonal status, tumour site (Glean 2001), and genetic factors (De Ruyck 2005). Extrinsic factors include the dose, volume and fraction of radiation, radiosensitisers, concurrent chemotherapy, and the site of treatment. Porock's theoretical framework of radiation skin-induced reactions (Figure 1) has categorised these factors into three broad categories: radiation therapy, genetic, and personal factors (Porock 2002).

Figure I. Porock's theoretical framework of radiation skin induced reactions (Porock 2002) (reprinted with permission).

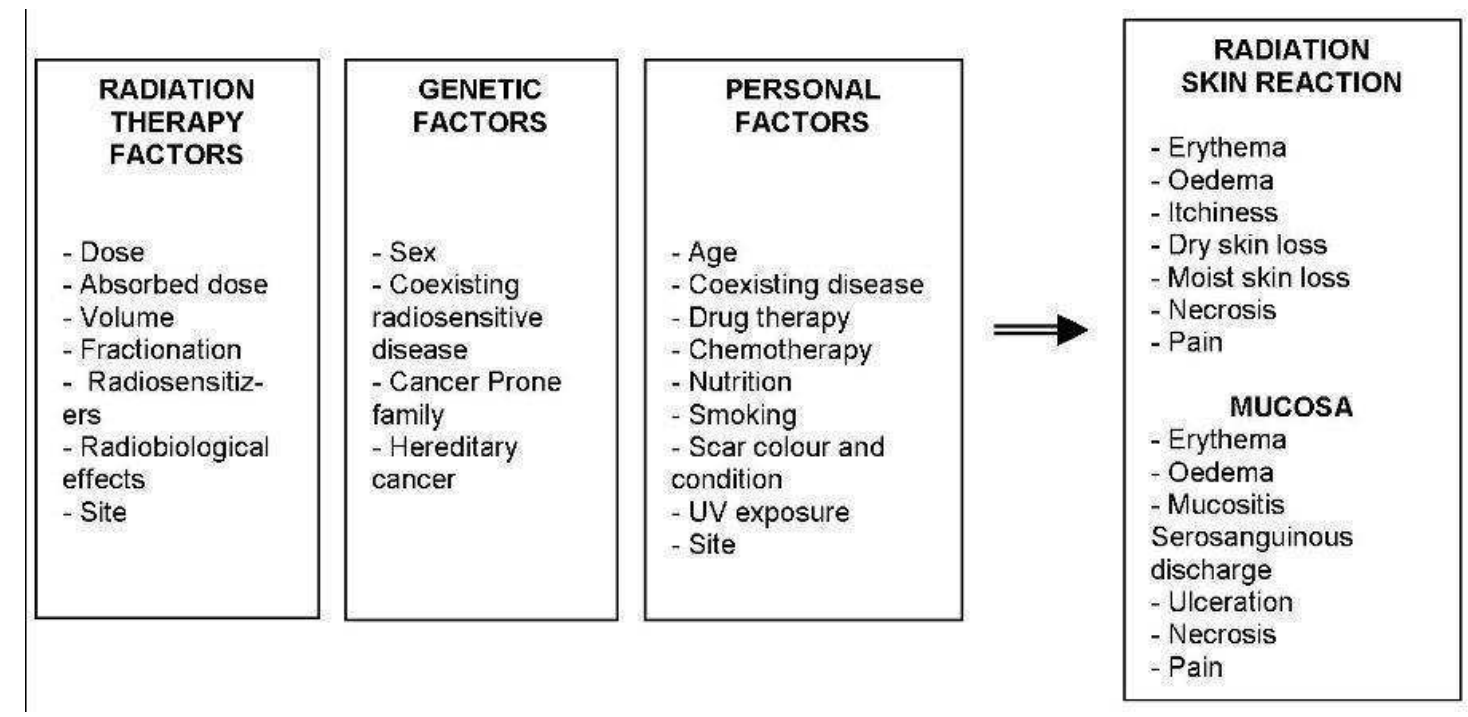

\section{Description of the intervention}

Interventions can be generally viewed as either preventive or management strategies (Bolderston 2002). Preventive strategies may include minimising irritants or irritations to the irradiated skin such as those associated with particular hygiene regimens, minimising friction, reducing the frequency of washing, avoiding the use of soap, cream and deodorants, and avoiding sun exposure
(Sitton 1992a; Wells 2003). Management strategies for established reactions may include active management of any reddening of the skin (erythema), any dry or moist shedding of the skin (desquamation), and ulceration of the skin, with topical preparations and dressings (Bolderston 2002; Glean 2001). Erythema is defined as the redness caused by flushing of the skin due to dilatation of the blood capillaries in the dermis (McFerran 1998). Dry desqua- 
mation is the shedding of the outer layers of the skin and moist desquamation occurs when the skin thins and then begins to weep as a result of loss of integrity of the epithelial barrier and a decrease in pressure exerted by plasma proteins on the capillary wall (Huda 2009).

\section{How the intervention might work}

\section{Preventive strategies}

There is both a lack of consensus amongst clinicians and scientific theories in the preventive strategies suggested to people receiving radiation therapy for their cancer. It is speculated that preventive strategies such as minimising irritants to the irradiated skin may help ensure that the outer keratinised (skin) surface remains as intact as possible, however removing the outer layer by cleansing may increase the chance of the underlying stem cell pool regenerating sufficiently to reduce the likelihood of moist desquamation and keep the outer skin surface intact. It is also known that inflammation may play a role in mediating the erythematous response to radiation in the skin (Simonen 1998). Hence, infrequent cleaning and washing may reduce bacterial load by maintaining the outer surface of the skin and therefore preventing colonisation, from organisms such as Staphylococcus aureus, the causative agent in many staph-infections, thereby reducing potential superantigen-induced inflammation and the likelihood of developing a red skin reaction (Hymes 2006).

\section{Management (treatment) strategies}

A range of topical preparations and dressings are used for the prevention and management of RISR, including moisturising creams, antioxidants, anti-inflammatories, alginate dressings, and hydrogel and hydrocolloid dressings. Each of these interventions are known to have different properties that aim to reduce the inflammation, contain fluid secretion, relieve discomfort, and/or to provide an optimum environment for wound healing (Glean 2001). In addition to those strategies identified above, it is possible that other interventions may include alternative medicine treatments such as traditional Chinese medicine, and herbal and/or dietary supplements. Nevertheless, there is currently insufficient documentation investigating how these interventions might work in people with RISR.

\section{Why it is important to do this review}

Radiation-induced skin reactions have an impact on the level of pain experienced and the quality of life of those who undergo radiotherapy treatment (Vaz 2007), and may even require changes to the person's radiation schedule (McQuestion 2006). In some cases, complex surgical reconstruction of damaged skin may be required (Cohn 2008). Therefore, managing skin reactions is an important priority in caring for those who undergo radiation therapy
(Noble-Adams 1999). Presently, a number of inconsistencies exist across radiation therapy centres with regard to the practice and recommendations given by health professionals to both prevent and manage this often painful side-effect of radiotherapy (Bolderston 2002; Boot-Vickers 1999; Glean 2001). There are several review papers within the literature in relation to management of RISRs (Glean 2001; Nystedt 2005; Richardson 2005).

A systematic review conducted by Bolderston and colleagues of the literature up to 2004 found that washing (which has paradoxically been reported as a risk factor for increasing skin reactions) was the only method that produced significant results in preventing skin reactions (Bolderston 2006). This suggestion currently contradicts advice to reduce washing which in turn may lead to cancer nurses doing more harm than good by avoiding washing the skin in order to prevent RISR (or vice versa). Although Bolderston's review provided some useful information, its scope was limited. For example, unpublished studies and trials reported in languages other than English were excluded, and it was unclear if experts in the field were contacted for any additional information. Moreover, no conclusion could be made regarding managing skin reactions with interventions other than washing. The review's outcomes were limited to levels of toxicity, pain, and itching, and excluded outcomes such as quality of life, levels of odour, and levels of participant satisfaction. In addition, the results of Bolderston's review did not report the effect size (odds ratio, relative risks), nor their confidence intervals. It is therefore our belief that a more comprehensive and updated systematic review is required in order to better inform practice of treatment of RISR and recommendations in this area.

In addition, we are also aware that a Cochrane systematic review by Kassab 2009 has investigated the effects of homeopathic medicines on side-effects of cancer treatment. This review focused on homeopathic medicines and therefore does not include any other strategies targeted by the current review.

\section{O B J E C T I VES}

To assess the effects of any interventions which aim to prevent or manage radiation-induced skin reactions in people with cancer.

\section{MET HO D S}

\section{Criteria for considering studies for this review}

\section{Types of studies}


All randomised controlled trials (RCT) providing a comparison between intervention types or a comparison between intervention and no intervention (usual care group) will be considered.

\section{Types of participants}

Participants will be those receiving radiation therapy, both external beam and brachytherapy (internal therapy treatments). There will be no restriction on age of the participants, diagnosis, previous or concurrent therapies, health status, dosage of treatment, location of irradiated area, or the setting where they receive their radiation treatment.

\section{Types of interventions}

Studies which compare an intervention with the aim of preventing or managing RISRs will be eligible. The inclusiveness of definitions of participants and interventions will ensure that this review is of use both to those in clinical practice as well as the wider population.

\section{Types of outcome measures}

\section{Primary outcomes}

We have made an a priori decision to analyse and discuss the outcomes for prevention studies and treatment studies separately. Although it may be possible to have outcomes that overlap prevention and treatment studies, we will reconcile these outcomes within the discussion section.

\section{Prevention studies}

(a) The development of a radiation-induced skin reaction (Yes/ No) (as measured by the assessment of the treating clinician or reported by participants).

\section{Treatment studies}

(b) Level of skin toxicity/reactions at one week and two weeks following the onset of the skin reaction (as measured by any instrument used by the author, such as the Radiation Therapy Oncology Group's (RTOG) acute radiation morbidity scale (Cox 1995)).

(c) Level of symptom severity at one week and two weeks following the onset of the skin reaction (physical or psychological) (as measured by any instrument used by the author).

\section{Secondary outcomes}

(a) Time taken to develop an erythema or dry desquamation (as measured by the assessment of the treating clinician, number of days since treatment commences) (from beginning to four weeks post-treatment).

\section{Treatment studies}

(b) Quality of life (as measured by any instrument used by the author such as WHOQOL-BREF (The WHOQOL Group 1998) (weekly during treatment up to one to two weeks post-treatment). (c) Time taken to heal (as measured by the assessment of the treating clinician, number of days since the onset of the RISR).

\section{Prevention and treatment studies}

(d) Cost of the interventions (both direct cost and indirect cost, both to the participant and to the health system).

(e) Participant satisfaction.

(f) Ease of use (as rated by the user of the intervention: participants or health professionals).

(g) Adverse effects. This may include allergic reactions (as reported in included studies).

\section{Search methods for identification of studies}

\section{Electronic searches}

We will search for relevant trials in the following:

- The Cochrane Skin Group Specialised Register;

- The Cochrane Central Register of Controlled Trials

(CENTRAL) in The Cochrane Library (latest issue);

- Medline OVID (from 2005 to present);

- EMBASE OVID (from 2007 to present);

- LILACS (Latin American \& Caribbean Health Sciences Literature, from inception to present);

- PsycINFO OVID (from inception to present);

- CINAHL EBSCO (1982 to present);

- Current Contents ISI (1998 to present); and

- Web of Science ISI (1966 to present).

The UK Cochrane Centre has an ongoing project to systematically search MEDLINE and EMBASE for reports of trials which are then included in the Cochrane Central Register of Controlled Trials. Searching has currently been completed in MEDLINE to 2004 and in EMBASE to 2006. Further searching will be undertaken for this review by the Cochrane Skin Group to cover the years that have not been searched by the UKCC.

We have devised a draft search strategy for RCTs for MEDLINE (OVID) which is displayed in Appendix 1. This will be modified to include additional search terms where necessary and will be used as the basis for search strategies for the other databases listed.

\section{Prevention studies}


We will search the following ongoing trials registers to identify ongoing or recently completed studies. If applicable, we will present relevant ongoing studies in a table in the review.

- The metaRegister of Controlled Trials

(www.controlledtrials.com).

- The U.S. National Institutes of Health ongoing trials register (www.clinicaltrials.gov).

- The Australian and New Zealand Clinical Trials Registry (www.anzctr.org.au).

- The World Health Organization International Clinical Trials Registry platform (www.who.int/ trialsearch).

- The Ongoing Skin Trials register (www.nottingham.ac.uk/ ongoingskintrials).

\section{Searching other resources}

\section{Reference Lists}

We will search the reference lists reported in any relevant reviews or other studies which were not identified via electronic searches. We will also scan the contents pages of relevant journals (since inception) for articles about interventions that aim at preventing or managing RSIRs and any abstracts from relevant conference proceedings. The relevant journals will include:

- Radiation Oncology;

- International Journal of Radiation Oncology;

- Biology and Physics, Radiotherapy and Oncology;

- Journal of Medical Imaging and Radiation Oncology;

- Journal of Pain and Symptom Management;

- Oncology Nursing Forum;

- Cancer Nursing;

- Journal of Alternative Therapies; and

- Journal of Integrative Cancer Therapies.

\section{Correspondence}

We will also contact authors of included studies for advice about other potentially relevant studies. We will also search the ProQuest Dissertations and Theses database for grey literature.

\section{Language}

There will be no restriction on language. Foreign language abstracts will be initially translated for the application of the inclusion and exclusion criteria, and where necessary the methods, results, and discussion sections will be translated for inclusion in the review. Translation of the abstract will be conducted by an appropriate Cochrane entity that has the capacity to do so.

\section{Data collection and analysis}

\section{Selection of studies}

Two review authors (RC and JW) will independently pre-screen all search results (titles and abstracts) for possible inclusion based on the inclusion criteria, and those selected by either or both authors will be subject to full-text assessment. These same two review authors will independently assess the selected articles against the inclusion criteria. Any discrepancies will be resolved by consensus, overseen by a third author (DB or BC) acting as arbiter. The arbiter will make the final decision for inclusion of the study. We will list those studies excluded after full-text assessment in the table 'Characteristics of excluded studies', and provide reasons for exclusion.

\section{Data extraction and management}

We will develop a data extraction form based on the Cochrane Skin Group's template, and pilot and amend it as necessary. The pilot of the data extraction form will include the first three eligible studies to facilitate data collection of any unexpected interventions in this area (if any). The first and second authors (RC and JW) will independently extract data using the data extraction form. Any errors or inconsistencies will be resolved. The first author (RC) will enter the data into RevMan, with JW checking the accuracy of data entry by independent data entry.

\section{Assessment of risk of bias in included studies}

We will assess and report on the risk of bias of included studies in accordance with the guidelines in the Cochrane Handbook for Systematic Reviews of Interventions (Higgins 2008), which recommends the explicit reporting of the following individual criteria:

(a) random allocation sequence generation;

(b) allocation concealment;

(c) blinding of participants, personnel, and outcome assessors (assessed for each main outcome or class of outcome);

(d) incomplete outcome data (assessed for each main outcome or class of outcome);

(e) selective outcome reporting (e.g. not reporting non-significant differences); and

(f) other sources of bias if noted.

We will also examine and report the following:

(g) validation and reproducibility of outcome measures;

(h) whether the study obtained ethics committee approval and ensured informed consent for participation; and

(i) use of standardised protocols for administration of preventive or management strategies.

Two review authors will independently assess the risk of bias in included studies, with any disagreements resolved by discussion and consensus, and with a third author acting as arbiter (either 
DB or BC). We will present our assessment in 'Risk of bias' tables for each included study. We will contact study authors for additional information about the study methods as necessary. We will incorporate the results of the risk of bias assessment into the review through narrative description and commentary about each of the items mentioned. This will lead to an overall assessment of the risk of bias of the included studies (Ryan 2007). We will assess each of the risk of bias items as 'yes' (indicating a low risk of bias), 'no' (a high risk of bias), and 'unclear' (risk of bias is unclear).

\section{Measures of treatment effect}

For individual trials, effect measures for dichotomous (binary) outcomes (i.e. whether the participant develops a RISR) will include relative risk (RR) with its $95 \%$ confidence interval (CI).

For outcomes with substantial effect size, number needed to treat (NNT) or number needed to harm (NNH), will be calculated.

For time-to-event data (i.e. time taken to develop RISR and time taken to heal) hazard ratios will be calculated.

For continuous outcomes (i.e. cost, levels of severity of RISR, levels of symptom severity, levels of participants' satisfaction), the effect measure will be mean difference (MD) or, if the scale of measurement differs across trials, standardised mean difference (SMD), each with its $95 \%$ CI. We acknowledge that this approach will only eliminate the differential due to the scale of measurement, but not that of a differential measurement sensitivity of the various instruments (e.g. different proneness to floor or ceiling effects).

For the levels of reaction/symptom severity, we will consider a $20 \%$ change in the measures to be clinically significant. This is because there are likely to be a diversity of tools used by trialists to measure levels of reaction/symptom severity and there may be a lack of information about the magnitude of their effect.

For meta-analyses and for dichotomous outcomes, risk ratios with their 95\% CI will be calculated; for categorical outcomes, estimates of RR with their $95 \%$ CI will be calculated; and for continuous outcomes, mean difference (MD) or a summary estimate for SMD, each with its 95\% CI, will be calculated. Data will be analysed using the Cochrane Collaboration's Review Manager 5 software.

\section{Unit of analysis issues}

Unit of analysis errors will be checked if cross-over trials or clusterrandomised trials are included. If required and sufficient data are available, we will recalculate the results using the appropriate unit of analysis (Higgins 2008).

To overcome a unit of analysis error for a study in relation to multiple treatment groups, we will combine all relevant experimental intervention groups of the study into a single group, and to combine all relevant control intervention groups into a single control group. This is to create a single pair-wise comparison (Higgins 2008).

\section{Dealing with missing data}

All analyses will be performed under intention-to-treat (ITT) principles, that is, participants will be analysed according to their allocated treatment groups irrespective of levels of compliance. We will also report on levels and note patterns of drop-outs in the intervention and comparison groups. Where there is an implication that studies have excluded data in their reported analyses, we shall contact authors for missing data. In the likelihood that, for most studies, some outcome data remain missing despite our attempts to obtain complete outcome data from authors, we will perform an available-case analysis, based on the numbers of participants for whom outcome data is known. If standard deviations (SD) are missing, where available, we shall compute them from standard errors using the formula $S D=S E x \sqrt{ } N$ (Higgins 2008). For the presentation of the main analyses, we shall not impute missing data from other studies, although to support our discussion of likely bias, we may consider sensitivity analyses incorporating best-case/ worst-case assumptions about the variability of SDs (guided by the range of included studies for any given analysis).

\section{Assessment of heterogeneity}

Heterogeneity will be tested using the $\mathrm{I}^{2}$ statistic (which describes the percentage of the variability in effect estimates that is due to heterogeneity rather than sampling error). A value greater than $50 \%$ will be considered to represent substantial heterogeneity ( Higgins 2008), and we will explore heterogeneity and possible reasons. We will undertake random-effects analyses as part of this.

\section{Assessment of reporting biases}

Reporting bias will be assessed using guidelines in the Cochrane Handbook for Systematic Reviews of Interventions (Higgins 2008). If enough studies are available to do a meaningful assessment of publication bias, a funnel plot will be constructed to test for asymmetry (this will be performed only for those primary outcome measures reported at the latest time points specified in the outcome section). We will discuss the results of the funnel plot and possible explanations for the results, which may include publication bias but also other sources of bias such as diverse methodological quality.

\section{Data synthesis}

A summary of the results of the data synthesis and assessment of the quality of the evidence will be included in a 'Summary of findings' table for the main comparison. Data will be grouped within the tables with respect to study design and type of intervention. We will separately present the results of studies that compare intervention with no intervention, then those that compare intervention strategies (e.g. hygiene regimen vs cream), and those that compare two or more types of strategies (e.g. hygiene regimen and 
steroidal cream vs hygiene regimen and moisturising cream). We will use this synthesis to prepare a narrative review of the results. This will be supplemented with a formal quantitative synthesis as detailed below where multiple similarly-designed studies are identified, to examine included studies to assess clinical and methodological heterogeneity. We will present the results of the studies as relative and absolute percentage excess of outcome in intervention compared to comparison group.

Although interventions may be classified as either preventive or management strategies (Bolderston 2002), we acknowledge that the interventions can be used for either or both purposes (Bolderston 2006). If included studies are sufficiently similar in terms of population, inclusion criteria, interventions and/or outcomes (including the time(s) at which these are assessed throughout the radiation regimen), we will consider pooling the data statistically using meta-analysis. A random-effects model is more likely to align with the expected heterogeneity of identified studies, although we shall simplify to a fixed-effect model if established to be appropriate at the point of analysis. These will report pooled mean differences (continuous variables using the same scale) or standardised mean differences (continuous variables measured on different scales), or risk ratios (dichotomous variables) and corresponding $95 \%$ confidence intervals. Numbers needed to treat (NNT) for benefit or harm will also be calculated where appropriate. For survival data, we will use hazard ratios for comparison in meta-analysis. If hazard ratios are not quoted in studies, then they will be calculated from available summary statistics (observed events, expected events, variance, confidence intervals, $P$ values, or survival curves) according to the methods proposed by Parmar and colleagues (Parmer 1998), or we will request these from authors. If cluster-randomised trials are included, we shall use the quoted design effects of clustering to adjust trial size to 'effective sample size' (i.e. sample size reflecting a simple, individual randomisation scheme); this will only be possible for studies that report their design effect or at least the outcome intra-class correlation coefficients. In the absence of these details, we shall assume design effects based, where available, on estimates from similar studies, or more likely perform sensitivity analyses applying a range of design effects based on group consensus of which outcomes might exhibit low (ICC = 0.005), medium (ICC = 0.02), and high (ICC $=0.05$ ) intra-cluster correlation coefficients. We will analyse separately the comparisons detailed in the previous paragraph. That is, separate meta-analyses will be carried out for each of the primary and secondary outcomes, and for subgroups of studies identified to be considering the same research question with similar designs.
The decision to carry out meta-analyses will be made by consensus of all authors.

\section{Subgroup analysis and investigation of heterogeneity}

If substantial heterogeneity ( $\mathrm{I}^{2}$ statistic $>50 \%$ ) exists between studies for the primary outcome we shall explore the reasons for heterogeneity; such as tumour site, dosage, treatment regimen (radiation therapy vs radiation therapy with concurrent chemotherapy vs radiation therapy with other adjuvant therapies) and treatment techniques such as three-dimensional conformal radiotherapy (3DCRT), and intensity-modulated radiation therapy (IMRT)/TomoTherapy.

We shall perform further subgroup analysis where adequate information is given. The groups will be tumour site and age of participants (children and adults) as described by the trial authors, treatment regimen, and treatment techniques.

\section{Sensitivity analysis}

We shall perform sensitivity analyses using all risk of bias components to assess low, moderate, or high risk of bias, where appropriate, in order to explore the influence of the following factors on estimates of effect size:

excluding unpublished studies and

excluding any small studies to establish how they impact on the results.

We will consider studies as having a low risk of bias if the study receives a 'Yes' rating for the criteria of sequence generation and allocation concealment.

\section{ACKNOWLEDGEMENTS}

The authors thank all those who have commented on the protocol throughout its development. In particular, we would like to thank Prof Hywel Williams (Co-ordinating Editor), Dr Finola Delamere (Managing Editor of the Cochrane Skin Group), and the editors and peer referees of the Cochrane Skin Group.

The Cochrane Skin Group editorial base would like to thank Jacques Bernier who acted as the clinical referee and Ann Fonfa who was the consumer referee. Thanks also to our statistical editor Jo Leonardi-Bee, our methods editor Philippa Middleton, and Hywel Williams who was the key editor on this protocol. 


\section{R E F E R E N C E S}

\section{Additional references}

\section{Bolderston 2002}

Bolderston A. Skin care recommendations during radiotherapy: A survey of Canadian practice. Canadian Journal of Medical Radiation Technology 2002; Vol. 34:3-11.

\section{Bolderston 2006}

Bolderston A, Lloyd NS, Wong RK, Holden L, Robb-Blenderman L. The prevention and management of acute skin reactions related to radiation therapy: A systematic review and practice guideline. Support Care Cancer 2006; Vol. 14, issue 8:802-17.

\section{Boot-Vickers 1999}

Boot-Vickers M, Eaton K. Skin care for patients receiving radiotherapy. Professional Nurse 1999; Vol. 14, issue 10:706-8.

\section{Camidge 2001}

Camidge R, Price A. Characterizing the phenomenon of radiation recall dermatitis. Radiotherapy and Oncology 2001;59:237-245.

\section{Cohn 2008}

Cohn AB, Lang PO, Agarwal JP, Peng SL, Alizadeh K, Stenson $\mathrm{KM}$, et al.Free-flap reconstruction in the doubly irradiated patient population. Plastic and Reconstructive Surgery 2008;122:125-32.

\section{Cox 1995}

Cox JD, Stetz J, Pajak TF. Toxicity criteria of the Radiation Therapy Oncology Group (RTOG) and the European Organization for Research and Treatment of Cancer (EORTC). International Journal of Radiation, Oncology, Biology, Physics 1995; Vol. 31, issue 5:1341-6.

\section{De Ruyck 2005}

De Ruyck K, Van Eijkeren M, Claes K, Morthier R, De Paepe A, Vral A, et al.Radiation-induced damage to normal tissues after radiotherapy in patients treated for gynecologic tumors: Association with single nucleotide polymorphisms in XRCC1, XRCC3, and OGG1 genes and in vitro chromosomal radiosensitivity in lymphocytes. International Journal of Radiation, Oncology, Biology, Physics 2005; Vol. 62, issue 4:1140-9.

\section{Glean 2001}

Glean E, Edwards S, Faithfull S, Meredith C, Richards C, Smith $\mathrm{M}$, et al.Intervention for acute radiotherapy induced skin reactions in cancer patients: The development of a clinical guideline recommended for use by the college of radiographers. Journal of Radiotherapy in Practice 2001; Vol. 2:75-84.

\section{Higgins 2008}

Higgins JPT, Green S. Cochrane Handbook for Systematic Reviews of Interventions. John Wiley \& Sons Ltd, 2008.

\section{Huda 2009}

Huda W. Review of Radiologic Physics. 3rd Edition. Balltimore: Lippincott, Williams \& Wilkins, 2009.

\section{Hymes 2006}

Hymes SR, Strom EA, Fife C. Radiation dermatitis: Clinical presentation, pathophysiology, and treatment 2006. Journal of the American Academy of Dermatology 2006; Vol. 54, issue 1:28-46.

\section{Kassab 2009}

Kassab S, Cummings M, Berkovitz S, van Haselen R, Fisher P. Homeopathic medicines for adverse effects of cancer treatments. Cochrane Database of Systematic Reviews 2009, Issue 2. [DOI: 10.1002/14651858.CD004845.pub2]

\section{Maduro 2003}

Maduro JH, Pras E, Willemse PH, de Vries EG. Acute and longterm toxicity following radiotherapy alone or in combination with chemotherapy for locally advanced cervical cancer. Cancer Treatment Reviews 2003; Vol. 29, issue 6:471-88.

\section{McFerran 1998}

McFerran TA. A Dictionary of Nursing. 3rd Edition. Cambridge: Oxford University Press, 1998.

\section{McQuestion 2006}

McQuestion M. Evidence-based skin care management in radiation therapy. Seminars in Oncology Nursing 2006; Vol. 22, issue 3: 163-73.

\section{Naylor 2001}

Naylor W, Mallett J. Management of acute radiotherapy induced skin reactions: A literature review. European Journal of Oncology Nursing 2001; Vol. 5, issue 4:221-3.

\section{Noble-Adams 1999}

Noble-Adams R. Radiation-induced reactions 1: An examination of the phenomenon. British Journal of Nursing 1999; Vol. 8, issue 17:1134-40.

Nystedt 2005

Nystedt KE, Hill JE, Mitchell AM, Goodwin F, Rowe LA, Wong FL, et al.The standardization of radiation skin care in British Columbia: A collaborative approach. Oncology Nursing Forum 2005; Vol. 32, issue 6:1199-205.

\section{Parmer 1998}

Parmar MK, Torri V, Stewart L. Extracting summary statistics to perform meta-analysis of the published literature for survival endpoints. Statistics in Medicine 1998;17(24):2815-2814.

Porock 2002

Porock D. Factors influencing the severity of radiation skin and oral mucosal reactions: Development of a conceptual framework. European Journal of Cancer Care (Engl) 2002; Vol. 11, issue 1: 33-43.

\section{Richardson 2005}

Richardson J, Smith JE, McIntyre M, Thomas R, Pilkington K. Aloe vera for preventing radiation-induced skin reactions: A systematic literature review. Clinical Oncology (Royal College of Radiologists) 2005; Vol. 17, issue 6:478-84.

\section{Ryan 2007}

Ryan R, Hill S, Broclain D, Horey D, Oliver S, Prictor M, Cochrane Consumers and Communication Review Group. Study Quality Guide. www.latrobe.edu.au/cochrane/resources.html (accessed 10 March 2009) 2007.

\section{Simonen 1998}

Simonen P, Hamilton C, Ferguson S, Ostwald P, O’Brien M, O'Brien P, et al.Do inflammatory processes contribute to radiation induced erythema observed in the skin of humans?. Radiotherapy 
and Oncology: Journal of the European Society for Therapeautic Radiology and Oncology 1998; Vol. 46, issue 1:73-82.

Sitton 1992

Sitton E. Early and late radiation-induced skin alterations. Part I: Mechanisms of skin changes. Oncology Nursing Forum 1992; Vol. 19 , issue 5:801-7.

\section{Sitton 1992a}

Sitton E. Early and late radiation-induced skin alterations. Part II: Nursing care of irradiated skin. Oncology Nursing Forum 1992; Vol. 19, issue 6:907-12.

The WHOQOL Group 1998

The WHOQOL-Group. Development of the World Health
Organization WHOQOL-BREF quality of life assessment. The WHOQOL Group. Psychological Medicine 1998; Vol. 28, issue 3: 551-8.

Vaz 2007

Vaz A, Pinto-Neto A, Conde D, Costa-Paiva L, Morais S, Esteves S. Quality of life of women with gynecologic cancer: Associated factors. Archives of Gynecology \& Obstetrics 2007; Vol. 276, issue 6:583-9.

\section{Wells 2003}

Wells M, MacBride S. Radiation skin reactions. Supportive Care in Radiotherapy. New York: Churchill Livingstone, 2003.

* Indicates the major publication for the study

\section{A P P E N D I CES}

\section{Appendix I. MEDLINE draft search strategy}

1. randomized controlled trial.pt.

2. controlled clinical trial.pt.

3. randomized.ab.

4. placebo.ab.

5. clinical trials as topic.sh.

6. randomly.ab.

7. trial.ti.

8. 1 or 2 or 3 or 4 or 5 or 6 or 7

9. (animals not (human and animals)).sh.

10. 8 not 9

11 exp Neoplasms/

12 exp Carcinoma/

13 (cancer* OR oncolog* OR neoplasm* OR carcinom* OR tumor* OR tumour* OR malignan* OR hematooncological OR hemato oncological OR hemato-oncological OR hematologic neoplasms OR hematolo* OR malignan*).mp.

14 (lymphom* OR T-cell OR B-cell OR sarcom* OR Ewing's OR Ewing* OR osteosarcom* OR wilms tumor OR wilms* OR nephroblastom* OR neuroblastom* OR rhabdomyosarcom* OR teratom* OR hepatom* OR hepatoblastom* OR PNET OR medulloblastom* OR PNET* OR neuroectodermal tumors, primitive OR retinoblastoma OR retinoblastom* OR meningiom* OR gliom* OR brain tumor* OR brain tumour* OR brain neoplasms OR central nervous system neoplasm* OR central nervous system tumor* OR central nervous system tumour* OR brain cancer* OR brain neoplasm* OR intracranial neoplasm* OR leukemia lymphocytic acute OR leukemia, lymphocytic, acute).mp.

15 (11 OR 12 OR 13 OR 14)

16 exp Radiotherapy/

17 exp Radiation Oncology/

18 (radioth\$ or radiat\$ or irradiat\$ or radiochemo $\$$ or chemoradi $\$$ ).mp.

19 (16 OR 17 OR 18)

20 Radiation induced skin reactions.mp.

21 (skin adj (reaction $\$$ or alter\$ or problem\$ or toxic\$ or care or change\$)).mp.

22 Dermatitis.mp.

23 Erythema

24 Desquamation

25 Itch

Interventions for preventing and managing radiation-induced skin reactions in cancer patients (Protocol)

Copyright (C) 2010 The Cochrane Collaboration. Published by John Wiley \& Sons, Ltd. 
26 Rash

27 (20 OR 21 OR 22 OR 23 OR 24 OR 25 OR 26)

28 (10 and 15 and 19 and 28)

\section{H I S T O R Y}

Protocol first published: Issue 5, 2010

\section{CONTRIBUTIONS OFAUTHORS}

Link with editorial base and co-ordinate contributions from co-authors (RC)

Draft protocol (All authors)

Developing the search strategies (RC, JW, DB, BC)

Run searches (RC)

Identify relevant titles and abstracts from searches i.e. broad screen (RC, JW)

Obtain full-text copies of trial reports (RC)

Selection of included trials (RC, JW, DB)

Extract data from trials (RC, JW)

Enter data into RevMan (RC)

Independent data entry (JW)

Carry out analysis (RC, JW, DB, BC)

Interpret analysis (RC, JW, DB, BC)

Draft final review (All authors)

Update review (All authors, RC will be the co-ordinating author)

\section{DECLARATIONS OF INTEREST}

None known.

SOURCES OF SUPPORT 


\section{Internal sources}

- Royal Brisbane and Women's Hospital, Australia.

- Royal Brisbane and Women's Hospital provided salary and facilities to RC and JW to conduct this systematic review.

- Institute of Health and Biomedical Innovation, Queensland University of Technology, Australia.

- Queensland University of Technology provided salary and facilities to DB to conduct this review.

- Division of Plastic Surgery, The Queen Elizabeth II (QEII) Health Science Centre, Halifax, Canada.

- QEII Health Science Centre provided salary and facilities to BC to conduct this review.

\section{External sources}

- No sources of support supplied 